\title{
Transcranial sonography: Brazilian experience
}

\author{
Sonografia transcraniana: uma experiência brasileira
}

José Luiz Pedroso', Edson Bor-Seng-Shu², Pedro Braga-Neto', Manoel Jacobsen Teixeira², Orlando Graziani

Povoas Barsottini ${ }^{1}$
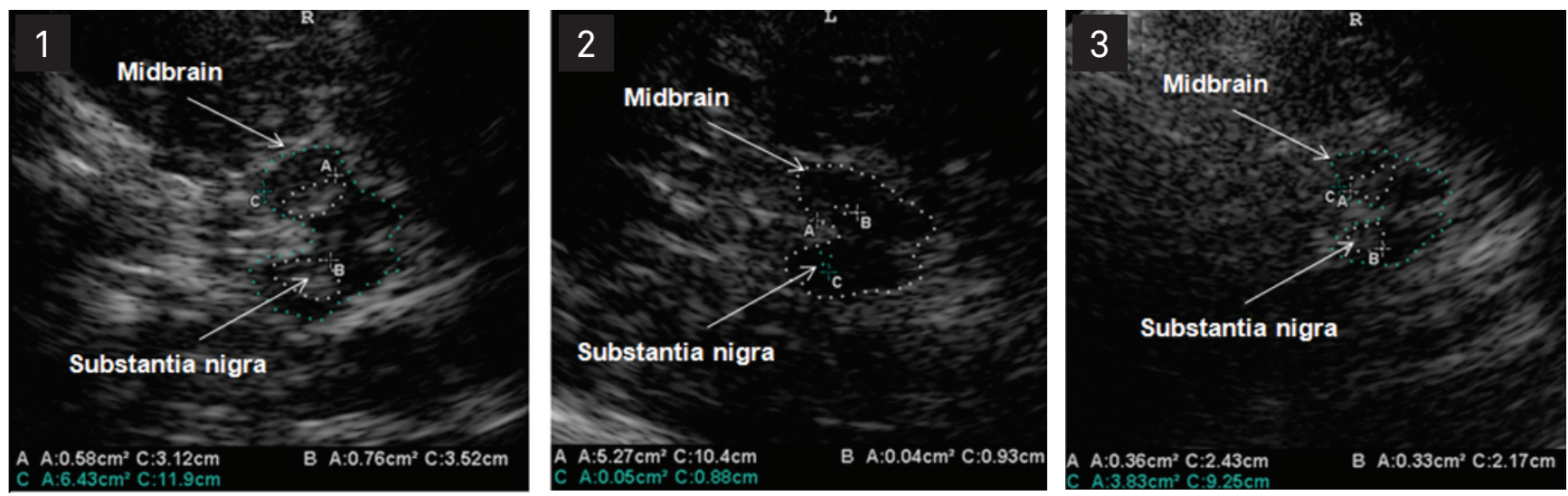

Figure. (1) Transcranial sonography (TCS) performed in a Parkinson's disease patient disclosing marked hyperechogenicity of the substantia nigra (SN); (2) TCS of a patient with idiopathic restless legs syndrome showing SN hypoechogenicity; (3) TCS of a spinocerebellar ataxia type 3 patient disclosing hyperechogenicity of the SN. The SN echogenic region was circled for better visualization. The hypoechogenic butterfly-shaped midbrain can be seen surrounded by hyperechogenic basal cisterns (midbrain was also encircled).

\section{Dear Editors,}

We have read with special interest the article by Fernandes et al. ${ }^{1}$ entitled "Transcranial sonography as a diagnostic tool for Parkinson's disease". These authors aimed to establish the percentage of subjects with adequated temporal bone windows for transcranial sonography (TCS) studies and also to revisit in a Brazilian sample the hypothesis that increased echogenic size of substantia nigra (SN) might distinguish Parkinson's disease (PD) patients from healthy individuals. We would like to congratulate the authors for the original idea to assess the Brazilian subjects with a case-control study. The authors found that $10.8 \%$ of the subjects had insufficient temporal acoustic bone windows, which is in line with previous data. Also, they identified hyperechogenicity of the SN in $88.2 \%$ of PD patients, against $14.2 \%$ of control group individuals.

In addition, the authors pointed out that "in Brazil there is no systematic study on transcranial sonography". Actually, this is an appropriate opportunity to report that our group has systematically studied TCS findings in some neurological diseases, such as spinocerebellar ataxias (SCA) and restless legs syndrome (RLS) (Figure). We have recently published a study entitled "Transcranial sonography findings in spinocerebellar ataxia type 3 (Machado-Joseph disease): a cross sectional study", in which we conducted TCS studies in 30 patients with clinical and molecular proven Machado-Joseph disease (MJD), and used 44 healthy subjects as control group, and found that hiperechogenicity of the SN was more frequent in MJD patients (75\%) than in the control group subjects (18\%), and that brain atrophy and lenticular nucleus hyperechogenicity occurred more frequently in MJD group. Two out of 30 MJD patients were excluded from the study due to limitation of acoustic temporal bone windows ${ }^{2}$.

Concerning atypical Parkinsonian syndromes, Barsottini et al. have already described an uncommon presentation of a Parkin heterozygous mutation carrier patient with a clinical phenotype resembling multiple system atrophy (MSA) (cerebellar form). TCS revealed SN hyperechogenicity, typically found in Parkin gene mutation carriers, and lentiform nucleus hyperechogenicity, described in MSA patients ${ }^{3}$. Also, Bor-Seng-Shu et al. reported the TCS findings of the first Brazilian PD case, who underwent deep brain stimulation of the subthalamic nucleus 4 .

We believe that TCS is a reliable, low-cost and noninvasive method for studying movement disorders, and efforts must be made to validate the literature findings in Brazilian population. 


\section{References}

1. Fernandes RCL, de Rosso ALZ, Vincent MB, et al. Transcranial sonography as a diagnostic tool for Parkinson's disease: a pilot study in the city of Rio de Janeiro, Brazil. Arq Neuropsiquiatr 2011;69:892-895.

2. Pedroso JL, Bor-Seng-Shu E, Felício AC, Braga-Neto P, Teixeira MJ, Barsottini OG. Transcranial sonography findings in spinocerebellar ataxia type 3 (Machado-Joseph disease): a cross-sectional study. Neurosci Lett 2011;504:98-101.
3. Barsottini OG, Felício AC, Aguiar PC, et al. Heterozigous exon 3 deletion in the Parkin gene in a patient with clinical and radiological MAS-C phenotype. Clin Neurol Neurosurg 2011;113:404-406.

4. Bor-Seng-Shu E, Fonoff ET, Barbosa ER, Teixeira MJ. Substantia nigra hyperechogenicity in Parkinson's disease. Acta Neurochir (Wien) 2010;152:2085-2087. 